\title{
Association between Pelvic Parameters and Vaginal Delivery
}

\author{
Tomohiro Yamada ${ }^{1}$, Yu Yamato ${ }^{1,2}$, Tomohiko Hasegawa ${ }^{1}$, Go Yoshida ${ }^{1}$, Tatsuya Yasuda ${ }^{3}$, \\ Tomohiro Banno ${ }^{1}$, Hideyuki Arima ${ }^{1}$, Shin Oe ${ }^{1,2}$, Yuki Mihara ${ }^{1}$, Hiroki Ushirozako ${ }^{1}$, \\ Koichiro Ide ${ }^{1}$, Yuh Watanabe ${ }^{1}$, Hironobu Hosino ${ }^{1}$, Yukihiro Matsuyama ${ }^{1}$ \\ ${ }^{1}$ Department of Orthopaedic Surgery, Hamamatsu University School of Medicine, Hamamatsu, Japan \\ ${ }^{2}$ Division of Geriatric Musculoskeletal Health, Hamamatsu University School of Medicine, Hamamatsu, Japan \\ ${ }^{3}$ Department of Orthopaedic Surgery, Iwata City Hospital, Iwata, Japan
}

\begin{abstract}
Study Design: Cross-sectional study.
Purpose: To investigate the association between vaginal delivery and pelvic parameters and clarify the effect of parity on parameter fluctuations.

Overview of Literature: During vaginal delivery, the sacroiliac joint widens and the sacrum nutates (nods). However, the association between these pelvic parameters and parity is unknown.

Methods: As part of a 2016 health screening, 320 female volunteers underwent whole-spine radiographs. Age-matched healthy women were grouped according to the number of vaginal deliveries $(0,1-2$, or $\geq 3)$. Demographic variables and spinopelvic parameters were compared among the three groups.

Results: Of the 320 volunteers, 213 were enrolled (mean age, $71.1 \pm 7.2$ years). The mean number of vaginal deliveries was 2.2. The average pelvic incidence (PI) was $55.6^{\circ} \pm 11.1^{\circ}$ and was significantly higher in the 90 women with three or more vaginal deliveries than in the other two groups $(p<0.001)$. The average sacral slope was $33.4^{\circ} \pm 11.1^{\circ}$ and was significantly higher in the women with three or more vaginal deliveries than in the 18 who did not deliver vaginally $(p<0.001)$. The 105 women with one or two vaginal deliveries had significantly higher Pls and sacral slopes than did those who did not deliver vaginally $(p<0.001)$.

Conclusions: This is the first study documenting an association between vaginal delivery and pelvic parameters. Bony birth canal realignment during vaginal delivery can affect postnatal PI. Our study helps in understanding the PI changes over a woman's life span.
\end{abstract}

Keywords: Vaginal delivery; Cesarean section; Pelvic incidence

\section{Introduction}

Pelvic incidence (PI), first defined by Legaye et al. [1], is the angle between the line perpendicular to the sacral endplate at its midpoint and a line connecting this point to the axis of the femoral head. PI, the sum of sacral slope and pelvic tilt [2], is fundamental for the regulation of pelvic spine parameters [3,4]. PI increases gradually during childhood as a result of walking on two legs [5]. After the bone matures, PI remains constant for every individual

Received Mar 16, 2021; Received Mar 16, 2021; Accepted Apr 9, 2021

Corresponding author: Tomohiro Yamada

Department of Orthopedic Surgery, Hamamatsu University School of Medicine, 1-20-1, Handayama, Higashi-ku, Hamamatsu-city, Shizuoka 431-3192, Japan

Tel: +81-53-435-2299, Fax: +81-53-435-2296, E-mail: cordial27@gmail.com 
in adulthood if sacroiliac joint movement does not occur [6]. However, during vaginal delivery, the sacroiliac joint might widen [7]. A well-known effect of estrogen is the proper ligamentous relaxation around the pelvis for delivery [8]. The estrogen concentration increases during pregnancy and peaks in the third trimester [9]. According to a previous study, during pregnancy, the strain force on the lumbar facet joints is more than double the average load [10], and the risk of degenerative spondylolisthesis increases with increasing parity [11]. A recent report from a database in the United Kingdom demonstrated that PI and lumbar lordosis mismatch increases with increasing parity [12].

By contrast, the association between pelvic parameters and vaginal delivery, during which bony changes in the birth canal occur, remains unknown. Thus, the major objective of this study was to use cohort analysis to investigate the association between vaginal delivery and pelvic parameters. This is the first study to elucidate the relationship between vaginal delivery and pelvic parameters.

\section{Materials and Methods}

\section{Subjects}

Subjects were community-dwelling volunteers who attended a basic local government health checkup in 2016. The checkup service includes musculoskeletal examination with whole-spine radiography and is conducted once every 2 years in Toei, Japan; most residents of middle and advanced age participate in the service [13-18].

Toei is in Aichi prefecture, in the middle of Japan, and has a population of 3,200 , of whom $49.6 \%$ are older than 65 years. Toei is in a relatively rural area, and many residents work in the agriculture and forestry industries.

In this study, we examined the distribution of vaginal deliveries by using a questionnaire. Background characteristics, including age, body mass index, bone mineral density, and spinopelvic parameters, were assessed. Exclusion criteria were (1) failure to return the questionnaire, (2) grade 2 or 3 vertebral fracture (semiquantitative) [19], (3) lumbarization or sacralization, (4) unclear radiographic images, and (5) a history of hip, spine, or abdominal surgery.

This study was approved by the Institutional Review Board of Hamamatsu University School of Medicine (IRB approval no., 15-060). Because of the retrospective nature of the research, the requirement for informed consent was waived.

\section{Radiographic assessment}

Whole-spine radiographs (anteroposterior and lateral) were taken while patients were standing. Lateral radiographs were obtained with patients gazing horizontally in a natural free-standing posture with their fingers on their clavicles [20]. All digitized radiographs were transferred to imaging software (Surgimap; Nemaris Inc., New York, NY, USA). The measured radiographic parameters were as follows: (1) lumbar lordosis (the angle between the upper endplate of the L1 vertebra and the upper endplate of the S1 vertebra), (2) thoracic kyphosis (the angle between the upper endplate of T5 and the lower endplate of T12), (3) sacral slope, (4) pelvic tilt, (5) PI, and (6) the distance between a plumb line from the center of the $\mathrm{C} 7$ vertebra and the superior-posterior center of the sacrum, or sagittal vertical alignment (C7 SVA). The same cohort data were used in an earlier study to determine reference values for spinopelvic parameters [21].

\section{Statistical analysis}

Descriptive statistics, including means and standard deviations, were calculated from demographic data and radiographic parameters. Differences in individual parameters, based on age or radiological data, were assessed via oneway analysis of variance according to the post hoc TukeyKramer test. All statistical computations were conducted using the IBM SPSS software ver. 22.0 (IBM Corp., Armonk, NY, USA), and a $p$-value of $<0.05$ was considered statistically significant.

\section{Results}

Of the 320 women who volunteered for the study, 60 were excluded on the basis of the exclusion criteria (Fig. 1). We then excluded 47 patients who were younger than 39 or older than 81 years. The 213 (81.9\%) who were included were classified into three groups according to the number of vaginal deliveries (none, one or two, or three or more) (Fig. 2). The ages of the women ranged from 48 to 80 years, and their mean age was $71.1 \pm 7.2$ years. The mean parity was 2.4 (range, $0-6$ ), and the mean number of vaginal deliveries was 2.2 (range, $0-6$ ). Of the participants, 90 
(42.3\%) had three or more vaginal deliveries, 105 (49.3\%) had one or two vaginal deliveries, and 18 (8.45\%) had delivered by cesarean section (CS).

Table 1 shows the demographic characteristics and spinopelvic parameters of the three groups. Age, body mass

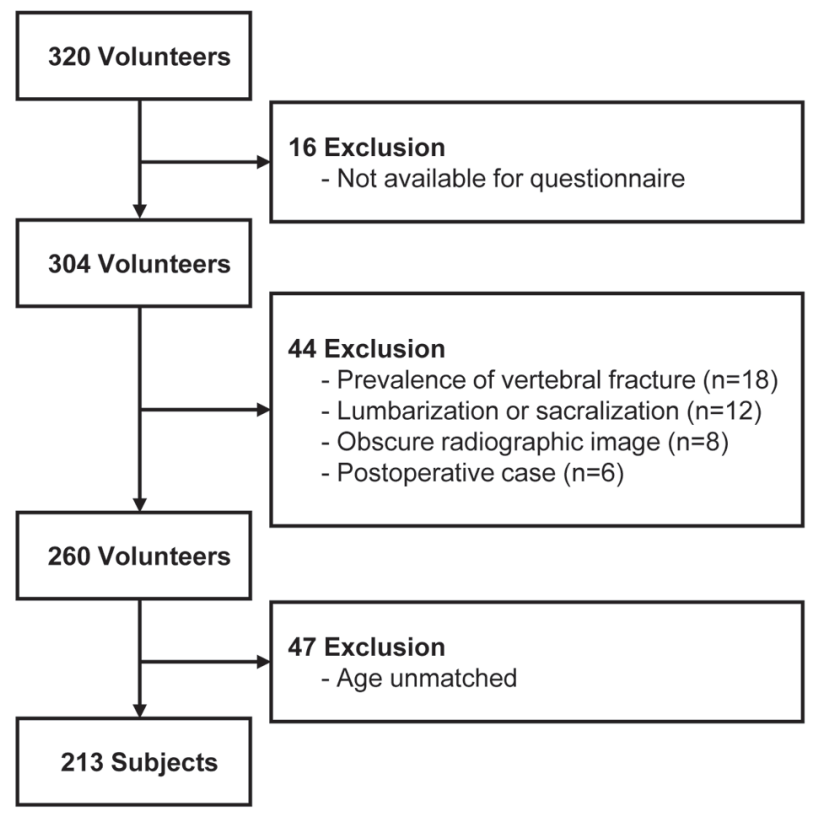

Fig. 1. Flow chart of subject selection and study design. index, and bone mineral density did not differ significantly among the three groups. Regarding spinopelvic parameters, PI was significantly higher in the women who delivered vaginally three or more times than in those who delivered vaginally once or twice and those who did not deliver vaginally $(p<0.001)$. Sacral slope was significantly higher in the women with three or more vaginal deliveries than in those with none $(p<0.001)$. PI-lumbar lordosis mismatch increased with the number of vaginal deliveries; however, the difference was not significant. Other spinopelvic parameters, including C7 SVA, thoracic kyphosis, and lumbar lordosis, did not differ significantly among the three groups. We measured all parameters with good reproducibility. For

\begin{tabular}{|c|c|c|}
\hline $\begin{array}{c}\text { P0 } \\
n=8\end{array}$ & \multicolumn{1}{c|}{$\begin{array}{c}\mathrm{P} 1-2 \\
\mathrm{n}=107\end{array}$} & $\begin{array}{c}\mathrm{P} \geq 3 \\
\mathrm{n}=98\end{array}$ \\
\hline \multicolumn{3}{|c|}{ CS } \\
$\mathrm{n}=10$
\end{tabular}

Fig. 2. Identification of vaginal delivery from whole parities. P, parity; CS, cesarean section; VD, vaginal delivery.

Table 1. Demographic characteristics and spino-pelvic parameters for the study participants

\begin{tabular}{|c|c|c|c|c|c|}
\hline \multirow{2}{*}{ Characteristic } & \multicolumn{3}{|c|}{ Vaginal delivery } & \multirow{2}{*}{$p$-value } & \multirow{2}{*}{ Post hoc } \\
\hline & $0(n=18)$ & $1-2(n=105)$ & $\geq 3(n=90)$ & & \\
\hline \multicolumn{6}{|l|}{ Demographic characteristics } \\
\hline Age (yr) & $70.2 \pm 8.5$ & $71.2 \pm 6.7$ & $71.1 \pm 7.0$ & 0.89 & \\
\hline Body mass index $\left(\mathrm{kg} / \mathrm{m}^{2}\right)$ & $22.9 \pm 3.4$ & $22.0 \pm 3.4$ & $22.6 \pm 2.9$ & 0.35 & \\
\hline Bone mineral density (\%YAM) & $80.4 \pm 15.2$ & $80.3 \pm 14.7$ & $83.9 \pm 17,3$ & 0.28 & \\
\hline \multicolumn{6}{|l|}{ Spino-pelvic parameters } \\
\hline Sagittal vertical axis (mm) & $19.0 \pm 41.5$ & $11.6 \pm 46.2$ & $6.9 \pm 41.2$ & 0.57 & \\
\hline Thoracic kyphosis $\left({ }^{\circ}\right)$ & $36.2 \pm 16.2$ & $33.7 \pm 12.1$ & $29.7 \pm 15.8$ & 0.088 & \\
\hline $\operatorname{LL}\left({ }^{\circ}\right)$ & $40.3 \pm 14.0$ & $46.4 \pm 16.8$ & $48.3 \pm 17.1$ & 0.27 & \\
\hline Sacral slope $\left({ }^{\circ}\right)$ & $24.4 \pm 8.8$ & $30.0 \pm 10.4$ & $33.4 \pm 11.1$ & 0.0055 & 0 vs. $\geq 3$ \\
\hline Pelvic tilt $\left({ }^{\circ}\right)$ & $20.8 \pm 8.6$ & $22.0 \pm 8.8$ & $22.2 \pm 9.9$ & 0.88 & \\
\hline $\mathrm{PI}\left({ }^{\circ}\right)$ & $45.1 \pm 8.6$ & $52.2 \pm 10.0$ & $55.6 \pm 11.1$ & 0.0014 & 0 vs. $\geq 3,1-2$ vs. $\geq 3$ \\
\hline $\mathrm{PI}-\mathrm{LL}\left({ }^{\circ}\right)$ & $4.7 \pm 14.1$ & $5.8 \pm 15.3$ & $7.3 \pm 16.0$ & 0.32 & \\
\hline \multicolumn{6}{|l|}{ Patient reported outcomes } \\
\hline Oswestry Disability Index (\%) & $14.2 \pm 11.7$ & $13.2 \pm 12.5$ & $11.3 \pm 11.3$ & 0.46 & \\
\hline
\end{tabular}

Values are presented as mean \pm standard deviation. Bold type is considered statistically significant. YAM, young adult mean; LL, lumbar lordosis; PI, pelvic incidence. 
Table 2. Demographic characteristics and spino-pelvic parameters in CS and VD subjects

\begin{tabular}{|c|c|c|c|}
\hline Characteristic & $\operatorname{CS}(n=18)$ & VD (n=105) & $p$-value \\
\hline \multicolumn{4}{|l|}{ Demographic characteristics } \\
\hline Age (yr) & $72.2 \pm 5.1$ & $71.2 \pm 6.8$ & 0.59 \\
\hline Body mass index $\left(\mathrm{kg} / \mathrm{m}^{2}\right)$ & $23.7 \pm 3.7$ & $22.1 \pm 3.2$ & 0.12 \\
\hline Bone mineral density (\%YAM) & $68.7 \pm 11.1$ & $72.9 \pm 13.4$ & 0.16 \\
\hline \multicolumn{4}{|l|}{ Spino-pelvic parameters } \\
\hline Sagittal vertical axis (mm) & $15.1 \pm 29.4$ & $11.5 \pm 45.2$ & 0.61 \\
\hline Thoracic kyphosis $\left({ }^{\circ}\right)$ & $37.7 \pm 14.5$ & $31.3 \pm 14.4$ & 0.40 \\
\hline $\operatorname{LL}\left({ }^{\circ}\right)$ & $44.1 \pm 11.8$ & $46.2 \pm 17.8$ & 0.24 \\
\hline Sacral slope $\left({ }^{\circ}\right)$ & $25.6 \pm 8.6$ & $31.1 \pm 10.9$ & 0.023 \\
\hline Pelvic tilt $\left({ }^{\circ}\right)$ & $20.1 \pm 7.4$ & $22.8 \pm 9.1$ & 0.43 \\
\hline $\mathrm{PI}\left({ }^{\circ}\right)$ & $45.9 \pm 8.1$ & $54.0 \pm 10.9$ & 0.0012 \\
\hline PI-LL $\left({ }^{\circ}\right)$ & $1.8 \pm 16.5$ & $7.9 \pm 17.1$ & 0.16 \\
\hline \multicolumn{4}{|l|}{ Patient reported outcomes } \\
\hline Oswestry Disability Index (\%) & $13.3 \pm 10.6$ & $12.8 \pm 12.1$ & 0.38 \\
\hline
\end{tabular}

Values are presented as mean \pm standard deviation. Bold type is considered statistically significant.

CS, caesarian section; VD, vaginal delivery; YAM, young adult mean; LL, lumbar Iordosis; PI, pelvic incidence.

C7 SVA, thoracic kyphosis, lumbar lordosis, sacral slope, pelvic tilt, and PI, the intraobserver intraclass coefficients were $0.913,0.996,0.926,0.936$, and 0.918 , respectively, and the interobserver intraclass coefficients were $0.991,0.990$, $0.964,0.962$, and 0.947 , respectively.

Table 2 shows the differences in demographic characteristics and spinopelvic parameters for women who delivered by CS and those who delivered vaginally. PIs were significantly higher in women who delivered vaginally than in those who delivered by CS $(p<0.001)$, as were sacral slopes $(p=0.023)$. PI-lumbar lordosis mismatch was greater in women who delivered vaginally than in those who delivered by CS; nevertheless, the difference was not significant. Other spinopelvic parameters, including C7 SVA, thoracic kyphosis, and lumbar lordosis, did not differ significantly between women who delivered vaginally than in those who delivered by CS.

\section{Discussion}

In this study, we aimed to examine the relationship between the number of vaginal deliveries and spinopelvic parameters. We found that women who delivered vaginally three or more times had the highest PIs among the

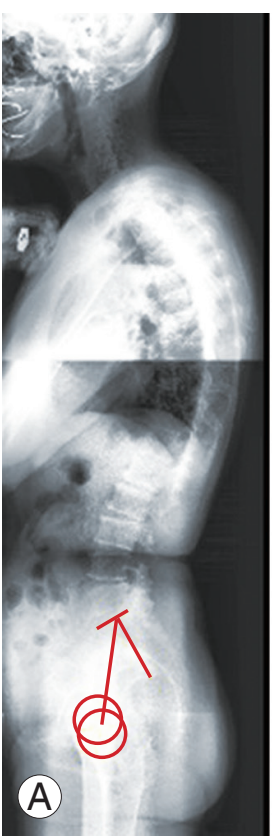

VD 0

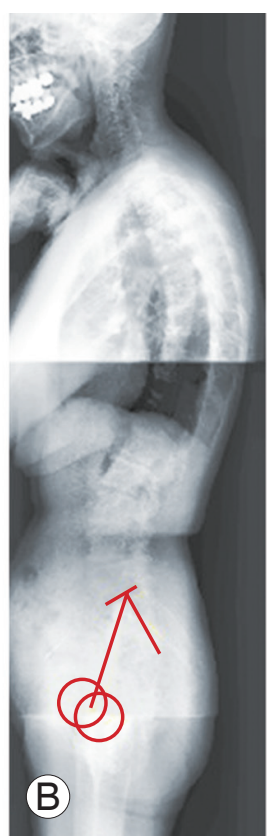

VD 1-2

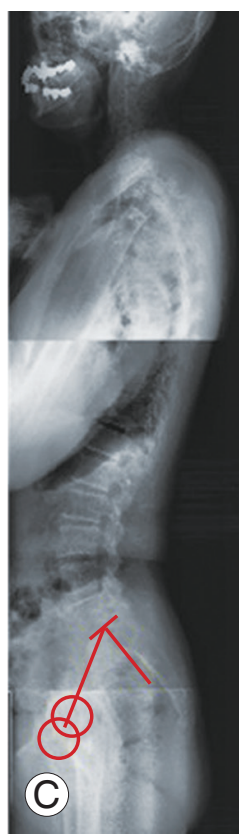

$\mathrm{VD} \geq 3$
Fig. 3. Representative pelvic incidence cases in each group: a 68-year-old woman without vaginal delivery (VD) (A); a 72-year-old woman with one vaginal delivery (B); and a 71-year-old woman with three vaginal deliveries (C)

three groups. Similarly, we demonstrated that women who delivered vaginally had higher PIs than did those who delivered by CS.

Multiparity has been reported as a predisposing factor for increasing PI-lumbar lordosis mismatch. Bailey et al. [12] reported that ligament laxity and biomechanical demands on the sacrum during pregnancy could change the PI. However, they did not find a significant difference in PI among groups classified according to parity. A possible explanation for this is that they could not determine the total number of vaginal and cesarean births. In that respect, our study shows the importance of evaluating the difference in PI according to vaginal delivery.

Vaughan [22] reported that the pelvic joints, including the sacroiliac joints and pubic symphysis, soften during pregnancy, and the true conjugate length increases by $0.5-1$ $\mathrm{cm}$ during vaginal delivery. Additionally, Borell and Fernstrom [23] reported that forward nutation of the sacrum occurred, causing pelvic expansion and enabling the passage of the fetus into the birth canal during vaginal delivery. The forward nutation of the sacrum results in increased sacral slope; therefore, increased PI could be attributed to the number of vaginal deliveries, during which fetus loading stress affects pelvis alignment, rather than the total 
parity (Fig. 3). Of interest is that we similarly observed that women who delivered vaginally had higher PIs than did those who delivered by CS (Table 2). Because bony birth canal movements are less likely to occur during CS, the PI differences according to whether subjects had delivered vaginally or not are valid measurements. The proportion of patients who delivered by CS in this study $(8.45 \%)$ is lower than the global incidence of CS delivery (31.2\%) [24]. Furthermore, sacral slope, in this study, was observed to be steeper than that of another cohort in Japan [25]; a possible explanation for this difference might be that a steeper sacral slope is associated with a broader birth canal, which allows women to deliver vaginally more easily.

This study had several limitations. First, we did not collect information about the ages of the women during each pregnancy. The expansion of the true conjugate line is greatest in younger pregnant women because aging increases the surface and number of ridges of the sacroiliac joint, which promotes interlocking and stability $[5,26]$. Therefore, it is uncertain whether women who give birth at a younger age eventually have a greater PI than those who deliver later in life. Second, we could not evaluate the effect of CS or at least one vaginal delivery on PI and sacral slope. Third, we could not determine whether a greater PI enables more efficient vaginal delivery. Thus, a longitudinal study would be needed to answer these questions adequately. However, our results provide information regarding the association between vaginal delivery and PI, since the participants were age-matched and none had any history of vertebral fracture or abdominal surgery.

\section{Conclusions}

Women who deliver vaginally have greater PIs than do women who deliver by CS. This is the first report demonstrating a correlation between vaginal delivery and PI.

\section{Conflict of Interest}

Yu Yamato and Shin Oe work at a donation-endowed laboratory in the Division of Geriatric Musculoskeletal Health. No potential conflict of interest relevant to this article was reported.

\section{Acknowledgments}

The authors would like to thank Keichi Nakai M.D. and Ms. Nao Kuwahara, secretaries of Department of Orthopedic Surgery, for their excellence in technical assistance during data collection.

\section{Funding}

The study was funded by the Medtronic Sofamor Danek Inc., Japan Medical Dynamic Marketing Inc., and Meitoku Medical Institution Jyuzen Memorial Hospital (funding sources of Yu Yamato and Shin Oe).

\section{Author Contributions}

Tomohiro Yamada wrote and prepared the manuscript, and all of the authors participated in the study design. All authors have read, reviewed, and approved the article.

\section{References}

1. Legaye J, Duval-Beaupere G, Hecquet J, Marty C. Pelvic incidence: a fundamental pelvic parameter for three-dimensional regulation of spinal sagittal curves. Eur Spine J 1998;7:99-103.

2. Mac-Thiong JM, Roussouly P, Berthonnaud E, Guigui P. Age- and sex-related variations in sagittal sacropelvic morphology and balance in asymptomatic adults. Eur Spine J 2011;20(Suppl 5):572-7.

3. Roussouly P, Pinheiro-Franco JL. Biomechanical analysis of the spino-pelvic organization and adaptation in pathology. Eur Spine J 2011;20(Suppl 5):60918.

4. Boulay C, Tardieu C, Hecquet J, et al. Sagittal alignment of spine and pelvis regulated by pelvic incidence: standard values and prediction of lordosis. Eur Spine J 2006;15:415-22.

5. Mac-Thiong JM, Berthonnaud E, Dimar JR 2nd, Betz RR, Labelle H. Sagittal alignment of the spine and pelvis during growth. Spine (Phila Pa 1976) 2004;29:1642-7.

6. Doi T, Tono O, Tarukado K, et al. A new sagittal parameter to estimate pelvic tilt using the iliac cortical density line and iliac tilt: a retrospective X-ray mea- 
surement study. J Orthop Surg Res 2015;10:115.

7. Garagiola DM, Tarver RD, Gibson L, Rogers RE, Wass JL. Anatomic changes in the pelvis after uncomplicated vaginal delivery: a CT study on 14 women. AJR Am J Roentgenol 1989;153:1239-41.

8. Samuel CS, Butkus A, Coghlan JP, Bateman JF. The effect of relaxin on collagen metabolism in the nonpregnant rat pubic symphysis: the influence of estrogen and progesterone in regulating relaxin activity. Endocrinology 1996;137:3884-90.

9. Van der Giessen J, Huang VW, van der Woude CJ, Fuhler GM. Modulatory effects of pregnancy on inflammatory bowel disease. Clin Transl Gastroenterol 2019;10:e00009.

10. Whitcome KK, Shapiro LJ, Lieberman DE. Fetal load and the evolution of lumbar lordosis in bipedal hominins. Nature 2007;450:1075-8.

11. Cholewicki J, Lee AS, Popovich JM Jr, et al. Degenerative spondylolisthesis is related to multiparity and hysterectomies in older women. Spine (Phila $\mathrm{Pa}$ 1976) 2017;42:1643-7.

12. Bailey JF, Sparrey CJ, Williams FM, Curran PF, Lotz JC, Kramer PA. The effect of parity on age-related degenerative changes in sagittal balance. Spine (Phila Pa 1976) 2020;45:E210-6.

13. Arima H, Dimar JR 2nd, Glassman SD, et al. Differences in lumbar and pelvic parameters among African American, Caucasian and Asian populations. Eur Spine J 2018;27:2990-8.

14. Yamato Y, Sato Y, Togawa D, et al. Differences in the geometrical spinal shape in the sagittal plane according to age and magnitude of pelvic incidence in healthy elderly individuals. J Orthop Sci 2020;25:55764.

15. Oe S, Togawa D, Nakai K, et al. The influence of age and sex on cervical spinal alignment among volunteers aged over 50. Spine (Phila Pa 1976) 2015;40:1487-94.
16. Yoshida G, Yasuda T, Togawa D, et al. Craniopelvic alignment in elderly asymptomatic individuals: analysis of 671 cranial centers of gravity. Spine (Phila Pa 1976) 2014;39:1121-7.

17. Ushirozako H, Yoshida G, Hasegawa T, et al. Impact of shift to the concave side of the C7-center sacral vertical line on de novo degenerative lumbar scoliosis progression in elderly volunteers. J Orthop Sci 2020;25:82-8.

18. Mihara Y, Togawa D, Hasegawa T, et al. Lumbar Retrolisthesis compensates spinal kyphosis. Spine Deform 2019;7:602-9.

19. Genant HK, Wu CY, van Kuijk C, Nevitt MC. Vertebral fracture assessment using a semiquantitative technique. J Bone Miner Res 1993;8:1137-48.

20. Faro FD, Marks MC, Pawelek J, Newton PO. Evaluation of a functional position for lateral radiograph acquisition in adolescent idiopathic scoliosis. Spine (Phila Pa 1976) 2004;29:2284-9.

21. Banno T, Hasegawa T, Yamato Y, et al. T1 pelvic angle is a useful parameter for postoperative evaluation in adult spinal deformity patients. Spine (Phila Pa 1976) 2016;41:1641-8.

22. Vaughan K. The expanding pelvis. Br Med J 1942;1:786.

23. Borell U, Fernstrom I. The movements at the sacroiliac joints and their importance to changes in the pelvic dimensions during parturtion. Acta Obstet Gynecol Scand 1957;36:42-57.

24. Roberts CL, Nippita TA. International caesarean section rates: the rising tide. Lancet Glob Health 2015;3:e241-2.

25. Funao H, Tsuji T, Hosogane N, et al. Comparative study of spinopelvic sagittal alignment between patients with and without degenerative spondylolisthesis. Eur Spine J 2012;21:2181-7.

26. Weisl $\mathrm{H}$. The articular surfaces of the sacro-iliac joint and their relation to the movements of the sacrum. Acta Anat (Basel) 1954;22:1-14. 